\title{
PERFIL SÓCIO DEMOGRÁFICO DOS PACIENTES ACOMETIDOS PELA COVID- 19
}

Social demographic profile of patients affected by Covid-19

Perfil social demográfico de pacientes afectados por covid-19

Loanda Carla Araújo de Medeiros ${ }^{1}(1)$; Maria da Conceição Alves Leal Borges ${ }^{2}{ }^{(1)}$; Mariana Pereira Gonsalves $^{3^{*}}{ }^{\circ}$; Gilberto da Costa Teodózio ${ }^{4}$ (1); Wesley Moura dos Santos ${ }^{5}{ }^{(0)}$

\section{Resumo}

A doença do coronavírus, trazida pelo vírus da covid-19, trouxe um impacto muito grande na vida das pessoas em todo o mundo pelo alcance e velocidade com o qual se disseminou (SOUZA, 2020). Segundo a Organização Mundial de saúde (2020), em 31 de dezembro de 2019 foram recebidas as primeiras notificações de casos de pneumonia com suspeita de serem provocados por uma nova cepa de Coronavírus, isso aconteceu na cidade de Wuhan na China. Diante disso, o estudo tem por objetivo traçar o perfil sócio demográfico dos pacientes acometidos pela COVID19, atendidos em um Hospital de Referência no Estado da Paraíba, apresentando por cada região os pacientes contaminados pela COVID 19. Trata - se de uma pesquisa transversal, descritiva e quantitativa, envolvendo indivíduos acometidos pela COVID 19, que foram atendidos no HMDJMP entre os meses de maio a dezembro de 2020. Este estudo pretendeu identificar, dentre os meses de maio a dezembro de 2020, o perfil sócio demográfico dos pacientes com diagnóstico positivo para COVID 19 atendidos no HMDJMP, bem como demarcar a microrregião com maior prevalência de pacientes regulados.

Palavras-chave: Coronavírus. Internações. Hospitalizações por Covid-19. Hospital Metropolitano Dom José Maria Pires.

\section{Abstract}

Coronavirus disease, brought on by the covid-19 virus, has had a huge impact in the lives of people around the world due to the range and speed with which it has spread (SOUZA, 2020). According to the World Health Organization (2020), on December 31, 2019, the first notifications of pneumonia cases with suspicion of being caused by a new strain of Coronavirus were received, this happened in the city of Wuhan in China. In view of this, the study aims to outline the socio-demographic profile of patients affected by COVID-19, seen at a Reference Hospital in the State of Paraíba, presenting patients infected by COVID 19 in each region. This is a cross-sectional survey, descriptive and quantitative, involving individuals affected by COVID 19, who were seen at the HMDJMP between May and December 2020. This study aimed to identify, between the months of May and December 2020, the socio-demographic profile of patients diagnosed positive for COVID 19 seen at the HMDJMP, as well as demarcating the micro-region with the highest prevalence of regulated patients.

Keywords: Coronavirus. Admissions. Hospitalizations by Covid19. Hospital Metropolitano Dom José Maria Pires.
1 Graduação em Enfermagem/Faculdade Santa Emília de Rodat (FAZER), Especialista em Urgência e Emergência Pré Hospitalar e Urgência e Emergência Intra Hospitalar pela Faculdade São Francisco da Paraíba.

2 Graduação em Enfermagem/Faculdade Santa Emília de Rodat (FAZER), Especialista em saúde coletiva com Ênfase em PSF/Faculdade Santa Emília de Rodat Paraíba Brasil; Especialista em Enfermagem em UTI adulto e Neonatal/Faculdade de Ciências Médicas e da saúde, SUPREMA, Minas gerais, Brasil.

3 Graduação em Enfermagem/Faculdade de Enfermagem São Vicente de Paula, Mestrado em Espiritualidade e Saúde/Universidade Federal da Paraíba, Especialista em Enfermagem Obstétrica/CEFAPP, Qualificação em Gestores do SUS/Fiocruz, Gestão do Trabalho e Educação na Saúde UFRN, Homeopatia - Instituto Holístico de Bioenergética, Paraíba, Brasil.

*Corresponding author: mari13gonsalves@gmail.com

4 Graduação em Enfermagem pelo Centro Universitário de João Pessoa (UNIPE), Membro da Comissão de Controle de Infeção Hospitalar (HMDJMP), Paraíba, Brasil.

5 Graduação em Enfermagem pela Faculdade Maurício de Nassau, Paraíba, Brasil. 


\section{Resumen}

La enfermedad del coronavirus, provocada por el virus covid-19, ha tenido un impacto muy grande en la vida de las personas en todo el mundo debido al alcance y la velocidad con la que se ha propagado (SOUZA, 2020). Según la Organización Mundial de la Salud (2020), el 31 de diciembre de 2019 se recibieron las primeras notificaciones de casos de neumonía sospechosos de ser causados por una nueva cepa de Coronavirus, esto sucedió en la ciudad de Wuhan, China. Por lo tanto, el estudio tiene como objetivo rastrear el perfil sociodemográfico de los pacientes afectados por COVID-19, atendidos en un Hospital de Referencia en el Estado de Paraíba, presentando pacientes infectados por COVID 19 para cada región. El estudio es descriptivo y cuantitativo, involucrando individuos afectados por COVID 19, que fueron atendidos en el HMDJMP entre los meses de mayo y diciembre de 2020. Este estudio tuvo como objetivo identificar, entre los meses de mayo y diciembre de 2020, el perfil sociodemográfico de los pacientes con diagnóstico positivo de COVID 19 atendidos en el HMDJMP, además de demarcar la microrregión con mayor prevalencia de pacientes regulados.

Palabras clave: Coronavirus. Admisiones. Hospitalizaciones por Covid-19. Hospital Metropolitano Dom José Maria Pires.

\section{Résumé}

La maladie à coronavirus, provoquée par le virus covid-19, a eu un impact très important sur la vie des gens dans le monde en raison de la portée et de la vitesse avec laquelle elle s'est propagée (SOUZA, 2020). Selon l'Organisation mondiale de la santé (2020), le 31 décembre 2019, les premières notifications de cas de pneumonie suspectés d'être causés par une nouvelle souche de coronavirus ont été reçues, cela s'est produit dans la ville de Wuhan, en Chine. Par conséquent, l'étude vise à tracer le profil sociodémographique des patients touchés par COVID-19, traités dans un hôpital de référence de l'État de Paraíba, présentant des patients infectés par COVID 19 pour chaque région descriptif et quantitatif, impliquant des individus touchés par COVID 19, qui ont été vus à l'HMDJMP entre les mois de mai et décembre 2020. Cette étude visait à identifier, entre les mois de mai et décembre 2020, le profil socio-démographique des patients avec un diagnostic positif pour COVID 19 vus au HMDJMP, ainsi que de délimiter la microrégion avec la prévalence la plus élevée de patients réglementés.

Mots-clés : Coronavirus. Admissions. Hospitalisations par Covid19. Hospital Metropolitano Dom José Maria Pires. 


\section{INTRODUÇÃO}

A doença do coronavírus, trazida pelo vírus da covid-19, trouxe um impacto muito grande na vida das pessoas em todo o mundo pelo alcance e velocidade com o qual se disseminou (SOUZA, 2020). Segundo a Organização Mundial de saúde (2020), em 31 de dezembro de 2019 foram recebidas as primeiras notificações de casos de pneumonia com suspeita de serem provocados por uma nova cepa de Coronavírus, isso aconteceu na cidade de Wuhan na China. Autoridades chinesas confirmaram se tratar de um novo tipo do vírus, o qual recebeu o nome de SARS-CoV-2 e devido à velocidade com a qual o vírus se espalhava entre os continentes, a OMS logo emitiu um alerta de emergência de saúde pública de importância internacional e exatamente em 11 de março de 2020, a situação é classificada, oficialmente, como uma pandemia.

Houve uma grande preocupação desde o início do novo surto de coronavírus (SARS-CoV-2) causador da COVID-19, em razão da alta taxa de transmissibilidade e com diferentes impactos, em várias regiões do mundo. Em virtude dessa elevada disseminação, tornou-se necessária a criação de um sistema para monitoramento dos casos da Covid-19 em tempo real em todo o mundo (LUDVIGSSON, 2020).

0 vírus se proliferou tão rapidamente que, em 18 de março de 2020, os casos confirmados da Covid-19 já haviam ultrapassado 214 mil em todo o mundo. No entanto, até aquele presente momento ainda não existia planos estratégicos prontos para serem aplicados e tentar conter a pandemia do novo coronavírus. Tem se discutido os sintomas comuns desta doença, visto que os dados atuais têm demonstrado que as confirmações do vírus variaram em pessoas assintomáticas ou com sintomas leves a pessoas gravemente doentes (PIMENTEL; DABOINI; OLIVEIRA; JR, 2020).

Já para o desenvolvimento de formas graves de COVID-19, tem-se observado vários fatores de risco identificados entre as populações. Contudo, os mais comuns, tem sido a idade avançada e a presença de comorbilidades, dentre as quais podemos citar as doenças cardíacas, doenças renais, diabetes, hipertensão, doenças respiratórias e crônicas. Na população em geral, a taxa de letalidade por COVID-19 varia entre $2-7 \%$, enquanto que nos doentes com mais de 80 anos, esses valores sobem para 15-20\%. (ONDER, REZZA, BRUSAFERRO, 2020). "As atuais taxas de letalidade poderão estar subestimadas, visto que uma proporção dos indivíduos infetados com SARS-CoV-2 não chega a ser diagnosticado, seja porque são assintomáticos e/ou não testados" (CARVALHO; COUTINHO; NUNES; MOURA; REGATEIRO, 2020)

Mediante os problemas, agravos e óbitos, ocasionados pela COVID-19 a toda população mundial, levantou-se o seguinte questionamento: qual é o perfil epidemiológico sócio demográfico dos pacientes acometidos pela doença do coronavírus atendidos no Hospital Metropolitano Dom José Maria Pires - HMDJMP? Diante disso, a realização dessa pesquisa, tem como relevância, o conhecimento do perfil sócio demográfico dos pacientes acometidos pela COVID-19, que foram atendidos no HMDJMP.

Esse perfil poderá contribuir para um entendimento epidemiológico dos indivíduos e assim, gestores e profissionais da saúde poderão planejar e tomar decisões com mais eficácia na execução das ações.

Desta forma, o estudo tem por objetivo traçar o perfil sócio demográfico dos pacientes acometidos pela COVID-19, atendidos em um Hospital de Referência no Estado da Paraíba, identificando a região com maior prevalência de pacientes acometidos pela COVID 19.

\section{METODOLOGIA}

Trata-se de uma pesquisa transversal, descritiva e quantitativa, envolvendo indivíduos acometido pela COVID 19, que foram atendidos no HMDJMP entre os meses de maio a dezembro de 2020. O estudo envolveu duas etapas metodológicas.

A primeira destinou-se à obtenção dos dados, extraídos a partir de uma análise documental e eletrônica de pacientes regulados pela Central Estadual de Regulação Hospitalar- CERH durante o período. Sendo incluídos na pesquisa todos os pacientes que positivaram para COVID 19 e excluídos aqueles que no momento da regulação se apresentaram com quadro clínico indefinido. Como instrumento de coleta dos dados, utilizamos informações, fornecidas pelo Núcleo Interno de Regulação - NIR COVID, por meio de planilha eletrónicaa de pacientes regulados pela CERH, 
acometidos e/ou suspeitos pela COVID 19, no HMDJMP.

A segunda etapa contemplou a ordenação e análise das informações obtidas na etapa anterior, onde os dados foram organizados em planilha, usando programas de computação. Em seguida, os resultados foram expressos pelo quantitativo e descrição das características socio demográficas dos pacientes que foram atendidos, sendo apresentados por meio de tabela. Foi calculada a frequência absoluta e relativa de todas as variáveis contidas no presente estudo e foi verificada a distribuição das características socio demográficas dos pacientes apresentadaa por cada região.

0 estudo foi realizado conforme os princípios éticos que constam na Resolução 466/2012 e 512/2016 do Conselho Nacional de Saúde e, por utilizar uma base de dados de acesso a identificação dos pacientes, foi necessária a aprovação do trabalho no Comitê de Ética em Pesquisa.

\section{RESULTADOS E DISCUSSÕES}

O estado da Paraíba é composto por 223 municípios, distribuídos por 12 microrregiões: Agreste da Borborema, Brejo Paraibano, Curimataú, Cariris Velhos, Catolé da Rocha, Piemonte da Borborema, Agro-Pastoril do Baixo Paraiba, Litoral Paraibano, Seridó Paraibano, Depressão do Alto-Piranhas, Sertão de Cajazeiras e Serra de Teixeira.

De acordo com os gráficos citados abaixo, podemos apresentar o quantitativo de solicitações de regulação/admissão, distribuídas por cidades a qual pertencem, como também os pacientes com suspeita para COVID 19, bem como pacientes positivos pelo vírus SARS-CoV-2, sendo estes, descartados do estudo.

Dessa forma, no período compreendido de maio a dezembro de 2020 apresentou-se por diversos municípios da Paraiba, a necessidade de atendimento especializado para pacientes acometidos pelo vírus SARS-CoV-2. Dentre eles, destacaram-se com maior prevalência : João Pessoa com 293(35,3\%), Santa Rita com 120(14,4\%), Guarabira com $112(13,5 \%)$, Itabaiana com $62(7,4 \%)$, Mamanguape com 47(5,6\%), Bayeux com 29(3,5\%), Cabedelo com 19(2,2\%), Sapé e Mari com 17 (2\%) cada, Pedras de Fogo com 14(1,6\%), Alhandra e Cruz do Espirito Santo com $8(0,9 \%)$ cada, Conde com $7(0,8 \%)$, Itapororoca e Solânea com $6(0,7 \%)$ cada, Caaporã e Pilar com 5(0,6\%) cada, Belém, Patos, Cajazeiras, Sousa e Ingá com 4(0,4\%), Pitimbu, Araruna, Jacaraú, Pombal, Rio Tinto e Conceição com 2(0,2\%) cada e Cacimba de Dentro, Campina Grande, Alagoinha, Lucena, São Miguel de Taipú, Lagoa de Dentro, Malta, Marcação, Paulista, Piancó, Picuí, Pocinhos, Queimadas, Salgado de São Félix, São Bento, Curral de Cima, Gurinhém, Igaraci, Juripiranga, Bom sucesso, Bonito de Santa Fé e Brejo do Cruz com 1(0,1\%). Diante desses dados, conseguimos excluir do presente estudo, pacientes oriundos das microrregiões da Serra de Teixeira, Cariris Velhos e Brejo Paraibano.

Gráfico1. Pacientes Regulados/Admitidos

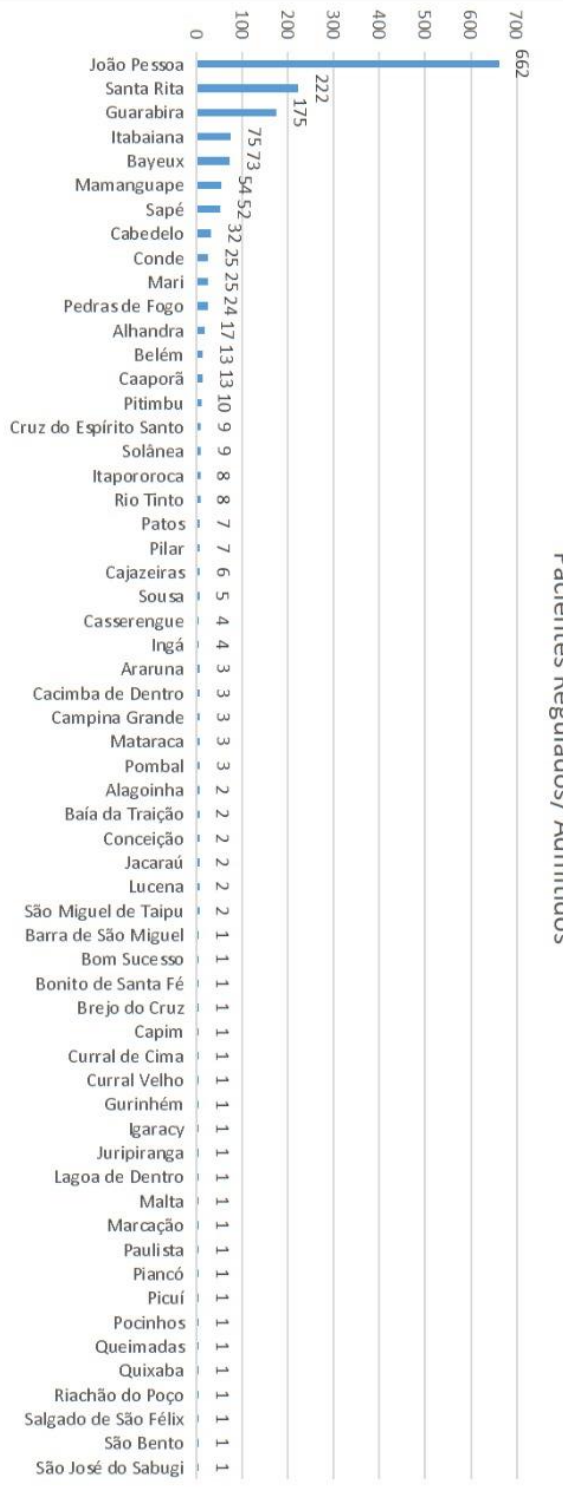

Fonte. Autores da Pesquisa, 2021. 
Gráfico 2. Pacientes com Suspeita de COVID-19.

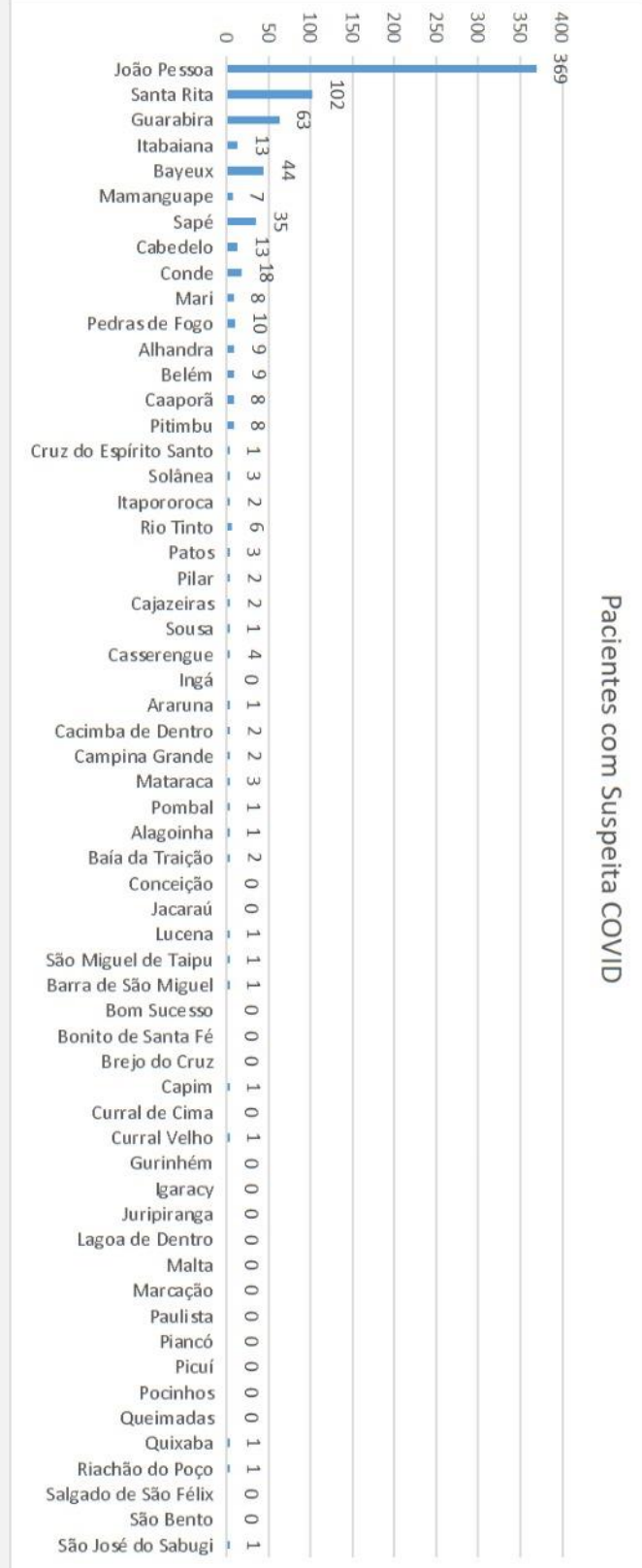

Fonte. Autores da Pesquisa, 2021.

Apresentou-se em maior prevalência de admissões, pacientes da microrregião do Litoral Paraibano, compreendendo 17 municipios (João Pessoa, Santa Rita, Mamanguape, Bayeux, Cabedelo, Pedras de Fogo, Alhandra, Cruz do Espírito Santo, Conde, Itapororoca, Caaporã, Pitimbu, Jacaraú, Rio Tinto, Lucena, Marcação e Curral de Cima) representando 565 admissões (68\%). Em sequência, seguimos com a microrregião Piemonte da Borborema, composto por 06 municípios (Guarabira, Belém, Ingá, Alagoinha, Lagoa de Dentro e Gurinhém), respondendo por 123 admissões (15\%). Seguimos para o Agro- Pastoril do Baixo Paraíba com 07 municípios (Sapé, Mari, Pilar,
Itabaiana, São Miguel de Taipú, Salgado de São Félix e Juripiranga) composto por 104 admissões (12,5\%).

Gráfico 3. Pacientes Positivos para COVID-19.

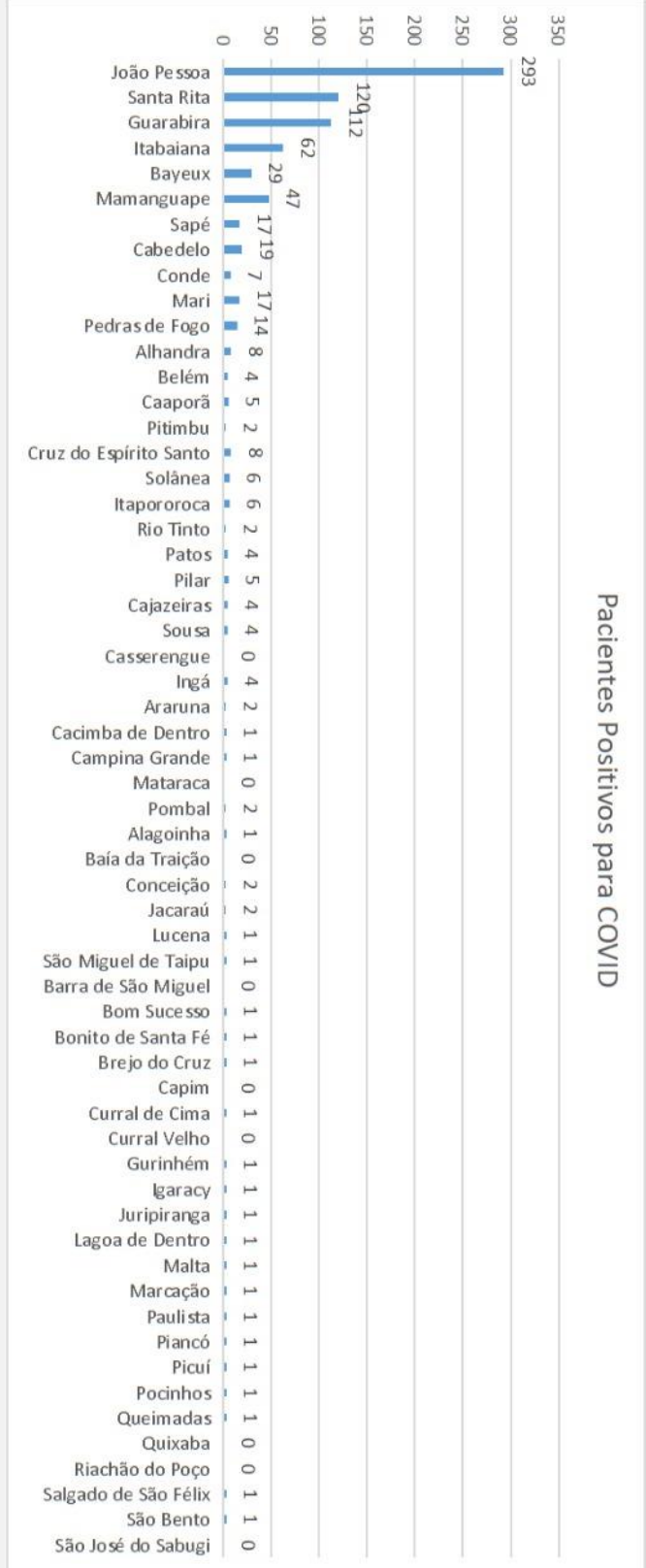

Fonte. Autores da Pesquisa, 2021.

Dando continuidade, prosseguimos com a microrregião Depressão do Alto Piranhas constituidos por 07 municípios (Patos, Sousa, Pombal, Paulista, Malta, Piancó e Igaraci) expressando 14 admissões (2\%). Chegando até a microrregião Agreste da Borborema, computamos 04 municípios (Solânea, Campina Grande, Pocinhos e Queimadas) com 09 admissões (1\%). Na sequência, temos as quatro últimas microrregiões: Sertão de Cajazeiras, composta por 03 municípios (Cajazeiras, Conceição 
e Bonito de Santa Fé) representando 07 admissões $(0,8 \%)$, Curimataú com 02 municipios (Araruna e Cacimba de Dentro) expresso por 3 admissões $(0,3 \%)$, Catolé do Rocha com 03 municipios (São Bento, Bom Sucesso e Brejo do Cruz) representando 03 admissões $(0,3 \%)$ e por último a microrregião do Seridó Paraibano composto por 01 município (Picuí) com apenas 01 admissão $(0,1 \%)$.

Com base no mesmo período, foram registrados 1.852 pedidos de regulação. Das 1.852 regulações, 1.589 pacientes foram admitidos e 263 não admitidos, tendo como justificativas: $57(22 \%)$ por óbito na origem, $124(47 \%)$ cancelados pela CERH, $31(12 \%)$ por desistência da família, 43(16\%) redirecionados para outros serviços e 08(3\%) evadiram-se da unidade de origem, conforme gráfico abaixo:

Gráfico 4. Regulações do NIR COVID.

Regulações NIR Covid

Admitidos/ Cancelados

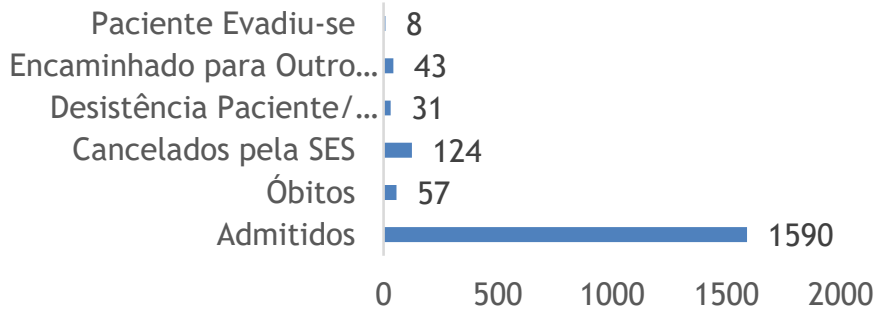

Fonte. Autores da Pesquisa, 2021.

Dos 1.589 pacientes admitidos, 829(52\%) chegaram com diagnóstico confirmado para Covid 19 e $760(48 \%)$ como suspeitos de acometimento pelo vírus. Analisando os 829 pacientes com diagnóstico confirmado para Covid 19, observou-se um quantitativo de $359(43 \%)$ pacientes do sexo feminino e $470(57 \%)$ do sexo masculino. Dessa forma apresenta concordância quando equiparado ao estudo de Niquine et. al (2020), onde relata haver uma prevalência de acometimentos por COVID 19 na população do sexo masculino quando em comparação com a população do sexo feminino.

Referente a divisão da população feminina por geração, de acordo com a época em que nasceram, comprovou-se a seguinte representatividade: pacientes com idade abaixo de 18 anos, representaram um quantitativo de $02(0,5 \%)$ pacientes, entre 19 a 30 anos um quantitativo de $10(2,5 \%)$ pacientes, entre 31 a 50 anos um quantitativo de $50(14 \%)$ pacientes, entre 51 a 70 anos um quantitativo de $133(37 \%)$ pacientes, entre 71 a 90 anos um quantitativo de $151(42 \%)$ pacientes e acima de 91 anos, 13(4\%) pacientes. Enquanto que, para população masculina, a representatividade por geração e de acordo com a época em que nasceram, apresentou-se da seguinte forma: pacientes com idade abaixo de 18 anos, representaram um quantitativo de $01(0,2 \%)$ pacientes, entre 19 a 30 anos um quantitativo de 19(4\%) pacientes, entre 31 a 50 anos um quantitativo de $96(20,4 \%)$ pacientes, entre 51 a 70 anos um quantitativo de $195(41,4 \%)$ pacientes, entre 71 a 90 anos um quantitativo de 147(31\%) pacientes e acima de 91 anos, 12(3\%) pacientes.

Com base nesses dados expostos, pôde-se constatar uma maior prevalência de acometimento para o vírus, pacientes do sexo masculino, na faixa etária compreendida entre 51 a 70 anos, seguidos de pacientes do sexo feminino na faixa etária entre 71 a 90 anos. Em subsequência seguimos com pacientes do sexo masculino entre 71 a 90 anos, pacientes do sexo feminino entre 51 a 70 anos, pacientes do sexo masculino de 31 a 50 anos, pacientes do sexo feminino de 31 a 50 anos, pacientes do sexo masculino de 19 a 30 anos, pacientes do sexo feminino acima dos 90 anos, pacientes do sexo masculino acima dos 90 anos, pacientes do sexo feminino entre 19 a 30 anos, pacientes do sexo feminino menores de 18 anos e por último pacientes do sexo masculino menores de 18 anos.

Estes pacientes foram acolhidos em áreas destinadas a pacientes com COVID de acordo com sua gravidade, distribuídos entre o Hospital de CampanhaHOSPITAL SOLIDÁRIO e setores COVID, sendo enfermaria e/ou UTIs situados dentro do HMDJMP. Difundidos por setores, foram admitidos no Hospital de Campanha 318(38\%) pacientes, Enfermaria COVID | 246(30\%) pacientes, enfermaria COVID || 22(2,5\%) pacientes, UTI COVID I 27(3\%) pacientes, UTI COVID II 155(19\%) pacientes, UTI COVID III 39(5\%) pacientes, UTI COVID IV 9(1\%) pacientes e UTI COVID 5 13(1,5\%). Conforme gráfico a seguir: 
Gráfico 5. Admissão e internação por setor.

Admissão por Local de Internação - Positivos

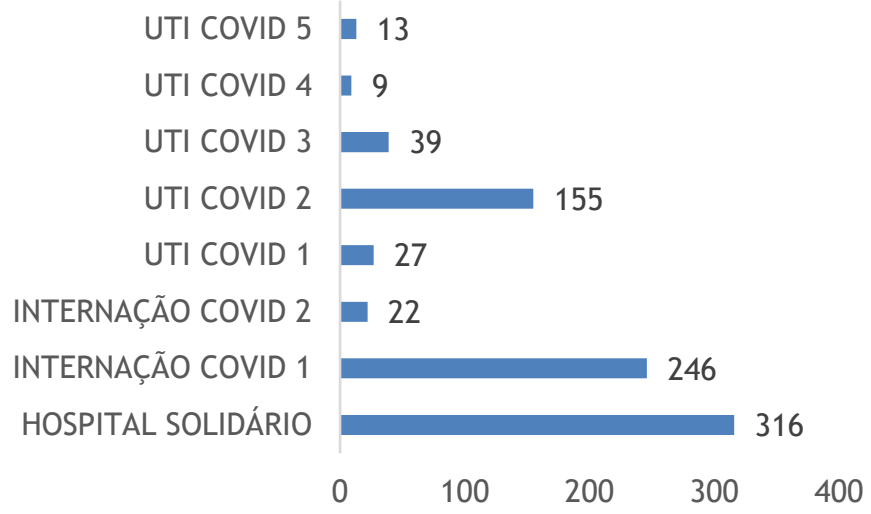

Fonte. Autores da Pesquisa, 2021.

\section{CONSIDERAÇÕES FINAIS}

Este estudo pretendeu identificar, dentre os meses de maio a dezembro de 2020, o perfil sócio demográfico dos pacientes com diagnóstico positivo para COVID 19 atendidos no HMDJMP, bem como demarcar a microrregião com maior prevalência de pacientes regulados. Ao final do estudo, conseguimos consubstanciar a microrregião do Litoral Paraibano, representada pela cidade de João Pessoa, como a mais recorrente tanto em solicitações quanto admissões, como também, identificamos a maior prevalência de pacientes positivo para COVID admitidos neste serviço, sendo do sexo masculino com idade compreendida entre 51 a 70 anos. Como contribuição, esperamos que este estudo venha a somar junto com a necessidade de mais estudos voltados para esta temática

\section{REFERÊNCIAS}

CARVALHO, J.C.; COUTINHO, I.A.; NUNES, I.; MOURA, A.L.; REGATEIRO, F.S. Asma e COVID-19: Atualização. Revista Portuguesa de Imunoalergologia, v. 28, n. 2, p. 97-109, 2020.

ONDER, G.; REZZA, G.; BRUSAFERRO, S. CaseFatality Rate and Characteristics of Patients Dying in Relation to COVID-19 in Italy. Jama, v. 323, n. 18, p. 1775-1776, 2020.
LUDVIGSSON J.F. Systematic review of COVID-19 in children shows milder cases and a better prognosis than adults. Acta paediatrica, v. 109, n. 6, p. 10881095, 2020. Disponível em: < https://onlinelibrary.wiley.com/doi/full/10.1111/a pa.15270> Acesso em: 28 de jun. 2021.

Organização Mundial da Saúde (OMS), Organização Pan-americana da saúde (OPAS). Folha informativa COVID-19 (doença causada pelo novo coronavírus). Disponível em: https://www.paho.org/bra/index.php?option=com content\&view=article\&tid $=6101$ : covid 19\&ltemid=875> Acesso em 27 de jan. 2021.

SOUZA, D.O. Pandemia de COVID-19 para além das Ciências da Saúde: reflexões sobre sua determinação social. Ciência \& Saúde Coletiva, v. 25, p. 24692477, 2020. Disponível em:< https: / /doi.org/10.1590/1413-

81232020256.1.11532020> Acesso em 10 de jan. 2021.

PIMENTEL, R. M.; DABOINI, B.E.G; OLIVEIRA, A. G. DE; JR, H. M. A disseminação da covid-19: um papel expectante e preventivo na saúde global. Journal of Human Growth and Development, v. 30, n. 1, p. 135, 2020. Disponível em< http: / / pepsic.bvsalud.org/scielo.php?script=sci_art text\&pid=S0104-

$12822020000100017 \& \operatorname{lng}=p t \& n r m=$ iso\&tlng=pt > Acesso em 25 de jan. 2021.

ALGO SOBRE, Regionalização da Paraíba: meso e microrregiões. Disponível em:<https://www.algosobre.com.br/geografia/regi onalizacao-da-paraiba-meso-e-microrregioes.html.> Acesso em 06 de abr. 2021.

NIQUINI, R.P.; LANA, R.M.; PACHECO, A.G.; CRUZ, O.G.; COELHO, F.C.; CARVALHO, L.M.; BASTOS, L.S. SRAG por COVID-19 no Brasil: descrição e comparação de características demográficas e comorbidades com SRAG por influenza e com a população geral. Caderno de Saúde Pública, 2020. Disponível

em:<https://www.scielo.br/j/csp/a/Zgn3w4jYm6n ZpCNt98K6Sdv/?format=pdf\&lang=pt> Acesso em 20 de Jul. de 20201 